\title{
REPORT ON THE THIRD WORKSHOP ON SUSTAINABLE SOFTWARE FOR SCIENCE: PRACTICE AND EXPERIENCES (WSSSPE3): APPENDICES
}

DANIEL S. KATZ(1), SOU-CHENG T. CHOI ${ }^{(2)}$, KYLE E. NIEMEYER ${ }^{(3)}$, JAMES HETHERINGTON ${ }^{(4)}$, FRANK LÖFFLER $^{(5)}$, DAN GUNTER ${ }^{(6)}$, RAY IDASZAK ${ }^{(7)}$, STEVEN R. BRANDT $^{(5)}$, MARK A. MILLER ${ }^{(8)}$, SANDRA GESING $^{(9)}$, NICK D. JONES ${ }^{(10)}, \operatorname{NIC~WEBER}^{(11)}$, SURESH MARRU ${ }^{(12)}$, GABRIELLE ALLEN $^{(13)}$ ', BIRGIT PENZENSTADLER ${ }^{(14)}$, COLIN C. VENTERS ${ }^{(15)}$, ETHAN DAVIS $^{(16)}, \operatorname{LORRAINE~HWANG}^{(17)}$, ILIAN TODOROV ${ }^{(18)}$, ABANI PATRA $^{(19)}$, MIGUEL DE VAL-BORRO $^{(20)}$

\begin{abstract}
This document contains three appendices to the report on the Third Workshop on Sustainable Software for Science: Practice and Experiences (WSSSPE3): Appendix A, The list of organizers; Appendix B, the list of attendees; and Appendix C, the list of travel award recipients.
\end{abstract}

\footnotetext{
${ }^{(1)}$ National Center for Supercomputing Applications (NCSA) \& Electrical and Computer Engineering (ECE) Department \& School of Information Sciences (iSchool), University of Illinois at Urbana-Champaign, IL, USA; d.katz@ieee.org.

(2) NORC at the University of Chicago and Illinois Institute of Technology, Chicago, IL, USA; sctchoi@uchicago.edu.

(3) School of Mechanical, Industrial, and Manufacturing Engineering, Oregon State University, Corvallis, OR, USA; kyle.niemeyer@oregonstate.edu.

(4) Research Software Development Group, University College London, UK; j.hetherington@ucl.ac.uk.

(5) Center for Computation \& Technology, Louisiana State University, Baton Rouge, LA, USA; $\{$ knarf,sbrandt\}@cct.lsu.edu.

(6) Lawrence Berkeley National Laboratory, Berkeley, USA; dkgunter@lbl.gov.

(7) RENCI, University of North Carolina at Chapel Hill, Chapel Hill, NC, USA; rayi@renci.org.

(8) University of California, San Diego, CA, USA; mmiller@sdsc.edu.

(9) Center for Research Computing, University of Notre Dame, Notre Dame, IN, USA; sandra.gesing@nd.edu.

(10) New Zealand eScience Infrastructure (NeSI), University of Auckland, Auckland, NZ; nick.jones@nesi.org.nz.

(11) University of Washington, Seattle, WA, USA; nmweber@uw.edu.

(12) Indiana University, Bloomington, IN, USA; smarru@iu.edu.

(13) National Center for Supercomputing Applications, University of Illinois at Urbana-Champaign, Urbana, IL, USA; gdallen@illinois.edu.

(14) College of Computer Engineering \& Computer Science, California State University, Long Beach, CA, USA; birgit.penzenstadler@csulb.edu.

(15) School of Computing and Engineering, University of Huddersfield, Huddersfield, UK; c.venters@hud.ac.uk.

(16) UCAR Unidata, Boulder, CO, USA; edavis@ucar.edu.

(17) University of California, Davis, CA, USA; ljhwang@ucdavis.edu.

(18) Science \& Technology Facilities Council, UK; ilian.todorov@stfc.ac.uk.

(19) Mechanical and Aerospace Engineering, University at Buffalo, Buffalo, NY, USA; abani@buffalo.edu.

(20) Department of Astrophysical Sciences, Princeton University, Princeton, NJ, USA; valborro@princeton.edu.
} 


\section{Appendix A. Organizing Committee}

The following is the list of organizers of WSSSPE3.

Daniel S. Katz

Gabrielle Allen

Neil Chue Hong

Sou-Cheng (Terrya) Choi

Sandra Gesing

Lorraine Hwang

Manish Parashar

Erin Robinson

Matthew Turk

Colin C. Venters
University of Chicago \& Argonne National Laboratory, USA

University of Illinois Urbana-Champaign, USA

Software Sustainability Institute. University of Edinburgh, UK

NORC at the University of Chicago \& Illinois Institute of Technology, USA

University of Notre Dame, USA

University of California, Davis, USA

Rutgers University, USA

Foundation for Earth Science, USA (local organizer)

University of Illinois Urbana-Champaign, USA

University of Huddersfield, UK

\section{Appendix B. Attendees}

The following is a list of participants registered for the WSSSPE3 workshop.

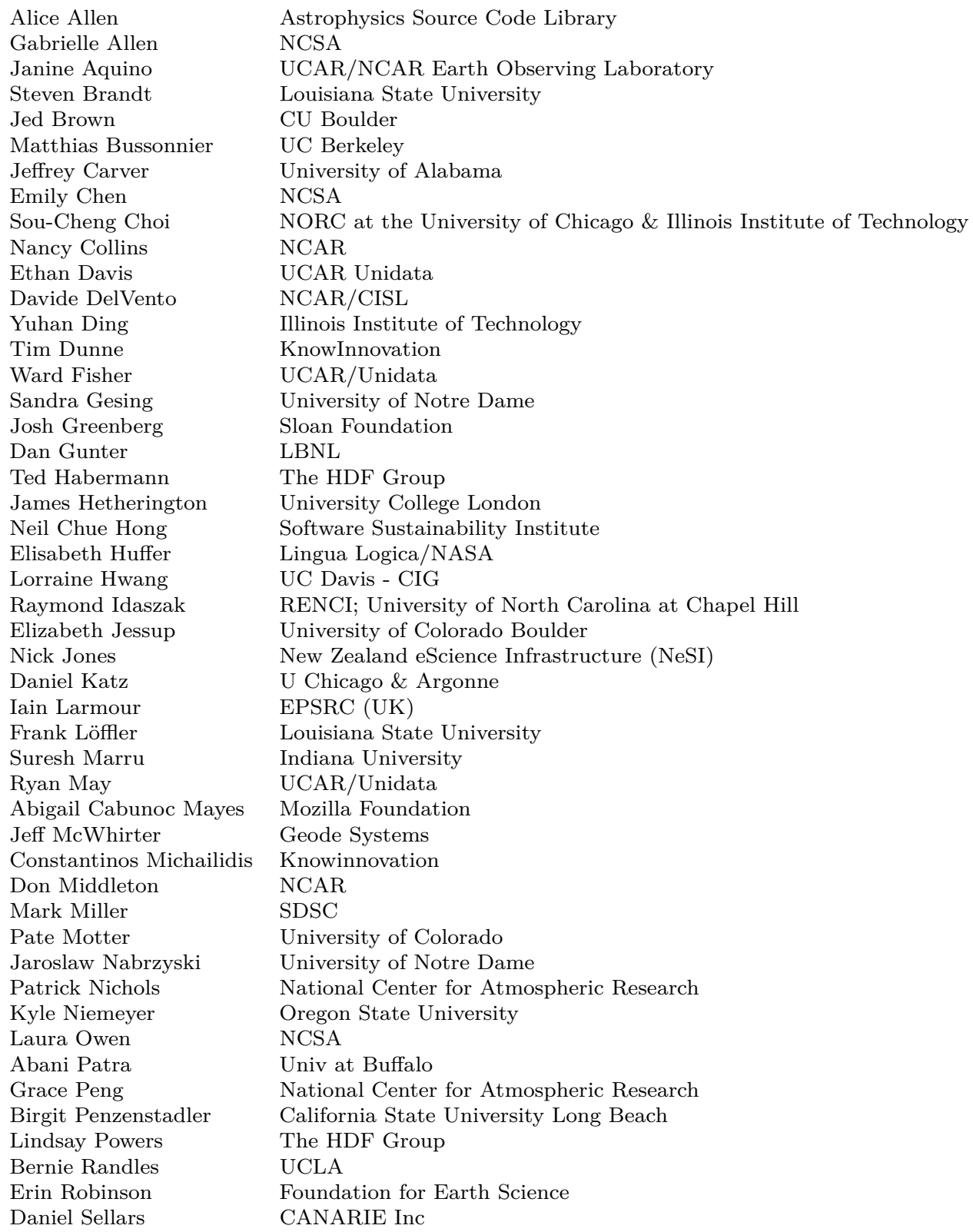




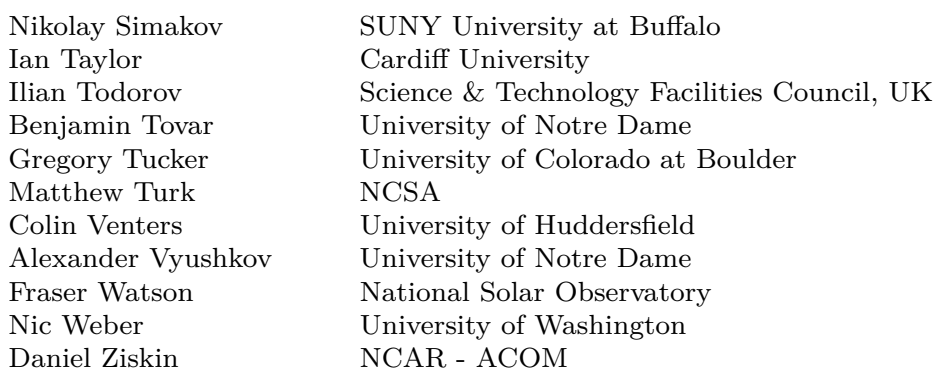

\section{Appendix C. Travel Award Recipients}

The following is the list of travel award recipients for the WSSSPE3 workshop.

Alice Allen
Steven Brandt
Jeffrey Carver
Emily Chen
Sou-Cheng Choi
Yuhan Ding
Lorraine Hwang
Ray Idaszak
Frank Löffler
Abigail Cabunoc Mayes
Pate Motter
Kyle Niemeyer
Birgit Penzenstadler
Bernadette Randles
Ilian Todorov
Nic Weber

Alice Allen

Lorraine Hwang

Abigail Cabunoc Mayes

Nic Weber

\author{
Astrophysics Source Code Library \\ Louisiana State University \\ University of Alabama \\ NCSA, University of Illinois \\ NORC at the University of Chicago \& Illinois Institute of Technology \\ Illinois Institute of Technology \\ CIG, UC Davis \\ RENCI, University of North Carolina at Chapel Hill \\ Louisiana State University \\ Mozilla Science Lab \\ University of Colorado \\ Oregon State University \\ California State University Long Beach \\ UCLA \\ STFC Daresbury Laboratory \\ University of Washington iSchool
}

